\title{
Color-Selective and Versatile Light Steering with up-Scalable Subwavelength Planar Optics
}

\author{
Giorgio Quaranta, ${ }^{\dagger, \ddagger \odot}$ Guillaume Basset, ${ }^{*},{ }^{\dagger}$ Olivier J. F. Martin, ${ }^{\ddagger \odot}$ and Benjamin Gallinet ${ }^{\dagger}$ \\ ${ }^{\dagger}$ CSEM SA, Muttenz Center, Muttenz 4132, Switzerland \\ ${ }^{\ddagger}$ EPFL Lausanne, Nanophotonics and Metrology Laboratory, Lausanne 1015, Switzerland
}

Supporting Information

\begin{abstract}
Resonant waveguide gratings (RWGs) are subwavelength structures of great interest for biosensors, optical filters and optical security applications. We demonstrate and characterize a beam steering device, where the incoupling and out-coupling processes make use of different RWGs that share the same ultrathin dielectric waveguide. This device enables selective color-filtering and redirection of a white light source (such as a white LED). Furthermore, this structure is compatible with up-scalable fabrication processes such as roll-to-roll replication, and is relevant for high-volume production. Because of its color selectivity and its use in low coherence illumination conditions, such a beam steering device

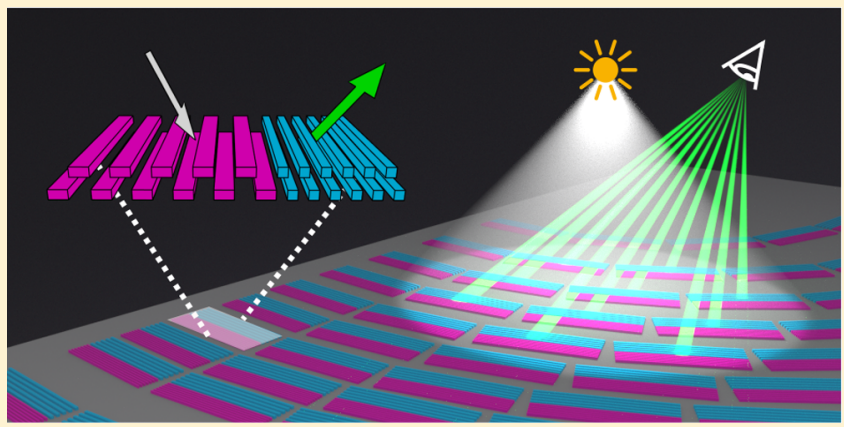
could be implemented in a variety of optical applications such as optical security, multifocal or monochromatic lenses, biosensors, and see-through optical combiners for near-eye displays.
\end{abstract}

KEYWORDS: planar optics, beam steering, white light source, up-scalable fabrication

Tn planar optics, beam steering techniques using micro- and nanostructuring surface are widely used for a variety of applications such as lasers, ${ }^{1}$ imaging systems, ${ }^{2,3}$ and sensing. ${ }^{4-8}$ Diffractive optical elements have also been largely implemented for beam shaping by realizing mathematically described arbitrary wavefronts. ${ }^{9}$ Diffractive optical elements are inherently designed to work with coherent sources, such as lasers, in given chromatic ranges, and they mainly work in paraxial approximation. Recently, metasurfaces (i.e., optically thin arrays of subwavelength spaced resonators $)^{10,11}$ have been created to steer and focus light, ${ }^{12-20}$ working in the visible range (390$750 \mathrm{~nm}$ ) or in part of it. ${ }^{21-28}$ The fabrication of these structures also requires high quality and low throughput ebeam lithography, making the realization of large samples very time-consuming. A few of the proposed metasurfaces are compatible with medium to high-throughput wafer-scale production techniques like steppers or those used in silicon technology. ${ }^{22,23,26-28}$ Moreover, none of these structures are compatible with very high-throughput fabrication techniques such as roll-to-roll nanoimprint lithography (NIL) replication, ${ }^{29}$ a requirement for many applications, such as optical security devices, ${ }^{30}$ where cost-effective processes are mandatory. ${ }^{31}$ Resonant waveguide gratings (RWGs) are structures that have been industrialized at very large volumes. ${ }^{32}$ RWGs were systematically analyzed in the 1990s. ${ }^{33-35}$ They enable an efficient transfer of wave energy between forward and backward propagations at resonance, by virtue of the relative phase shift between the incoming and the diffracted waves. Thanks to leaky propagation and accumulation in the RWGs, the guided wave they support produces stronger color selectivity compared to the localized energy of a standard resonator. However, using a RWG made with a single periodicy, the beam deflection occurs only at the zeroth order. ${ }^{36}$

Here, we propose a thin-film single-layer structure based on RWGs $^{35}$ that performs selective color-filtering and beam steering. It versatilely works in both reflection and transmission, with white light and low coherent source, without prefiltering. The fabrication of the master for the structure involves an efficient and fast ebeam lithography technique that allows the exposure of patterns larger than $1 \mathrm{~cm}^{2}$. The structure is replicated using NIL in a readily up-scalable process, such as roll-to-roll production, and therefore potentially relevant for very high-volume production. The proposed method allows to create a device that can arbitrarily filter and redirect portions of white light from one point source to another point. It is therefore possible to have, as a direct application, an image reproduced in a specific position that can be visualized with a smartphone, using the white LED flash as the low-coherent source and the camera the observer. We first present an analytical model of the structure, together with FDTD simulations. We then describe the up-scalable fabrication method used. Finally, we characterize a fabricated device designed to be an optical authentication feature working with standard smartphones.

Received: March 9, 2017

Published: May 1, 2017 
a

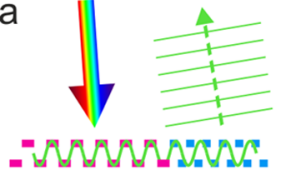

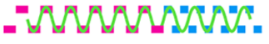

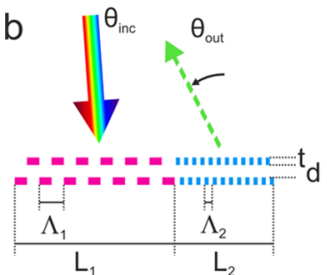

$\mathrm{C}$
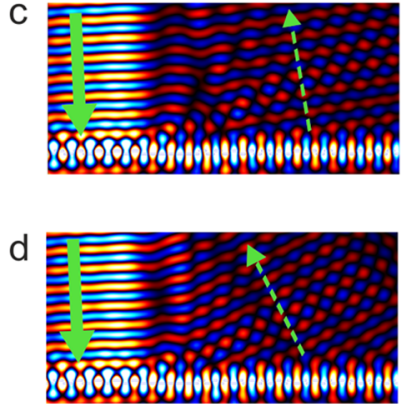

e

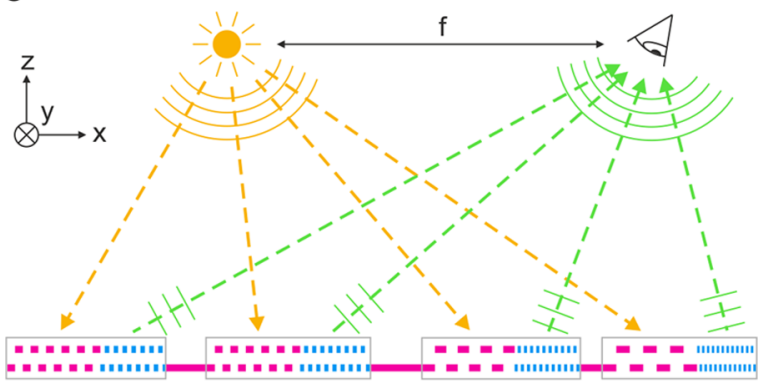

Figure 1. Schematic of a single unit cell showing the steering in reflection. (a) Corrugated ultrathin waveguide coats a first and a second adjacent gratings, schematized respectively in pink and blue. A specific wavelength range is in-coupled inside the waveguide by the first grating from a white incident light beam, and out-coupled from the second grating. (b) By changing the period of the second grating, it is possible to out-couple the light at a different in-plane angle. (c) and (d) Corresponding amplitude of the transversal near-field obtained with FDTD simulations. (e) It is possible to engineer a complete surface that redirects the light coming from one point to another using constructive interference, as represented here in the inplane regime.

The unit cell of the structure consists of two adjacent finitelength and coupled RWGs. A typical device is composed of a collection of unit cells, which schematic is shown in Figure 1a,b: two RWGs with periods $\Lambda_{1}$ and $\Lambda_{2}$ and lengths $L_{1}$ and $L_{2}$ are coupled together. Both resonant gratings share a thin highrefractive index (HRI) guiding layer, where the corrugated waveguide thickness $t$ is well below the wavelength, as the modulation depth $d$ of the supporting grating. Furthermore, for simplicity, we chose to have $L_{1}=L_{2}$ in order to have the boundary between the two RWGs in the center of the unit cell. However, it is possible to customize the ratio between $L_{1}$ and $L_{2}$ to enhance the efficiency of the structure, that is, the grating responsible for in-coupling could be longer than the grating for out-coupling. The broad-band light source incident on the first RWG can be partly coupled into leaky guided modes when the phase-matching of the propagation wave vector is fulfilled. ${ }^{34,35}$ In particular, various modes can propagate in the waveguide layer in different directions. Since the RWG has a finite-length $L_{1}$ the coupled modes have a spectral band whose width depends on the grating length. ${ }^{37}$ The coupled leaky modes propagating to the second RWG can be out-coupled if they are phase-matched with the resonance of the second RWG. ${ }^{35}$ Specifically, we demonstrate devices using very thin corrugated waveguides allowing only a few waveguide modes in the visible range. Therefore, the coupled leaky modes propagate in the first and later second RWG with a field distribution that extends into the cladding and the guided propagation is similar to the propagation in a noncorrugated waveguide having the same thickness $t$. Since the second RWG has the same corrugated thickness $t$, both RWGs are almost impedance-matched and there are low losses at their interface. The direction of the outcoupled modes differs from the direction of the incoming light because the periods of the two RWGs are different. It is possible to tune the direction of the out-coupled modes by changing the period of the second RWG, as represented in Figure $1 \mathrm{~b}$ in the in-plane regime. ${ }^{38}$ For beam steering out of the in-plane regime, the grating orientation and spatial periodicity of the two RWGs can be tuned as detailed below. For binary RWGs as in the case here or sinusoidal RWGs, the leaky-guided modes are out-coupled in both reflection and transmission with the same steered angles and almost the same efficiency. Since operation for such a device is usually in reflection, we omit in
Figure 1 the light transmitted downward. It should be mentioned that, in general, different spectral portions of the light source can be in-coupled into the second RWG, and be out-coupled from the first RWG. This structure supports only strong resonances for the light polarized transverse electric (TE), with the electric field parallel to the $y$-axis in Figure 1c. Resonances for the orthogonal, transverse magnetic (TM) polarization are negligible compared to those of TE modes in case of corrugated thin-film waveguides. ${ }^{39,40}$ This is confirmed by numerical simulations (data not shown). It is however possible extend this concept to thicker waveguides, to efficiently allow the operation in both polarizations.

Let us first derive an analytical model to describe the process of in-coupling and out-coupling for this structure, based on the phase matching condition for a slab waveguide. ${ }^{41}$ The model is valid when both the modulation depth $d$ and the waveguide thickness $t$ are well below the wavelength, such that the propagation behavior of the leaky modes can be modeled as a propagation in a noncorrugated waveguide. The model becomes inaccurate when $t, d$ are comparable to the effective wavelength. The model is very well suited for waveguides having thicknesses $t$ much below the wavelength used. We use this model to predict the grating periods (respectively, $\Lambda_{1}$ or $\Lambda_{2}$ ) that are needed to in-couple and to out-couple from the waveguide a central wavelength $\lambda$, using the light incidence and steering directions given in polar and azimuthal angles in the cladding material, $\left(\theta_{\text {in }}, \phi_{\text {in }}\right)$ and $\left(\theta_{\text {out }}, \phi_{\text {out }}\right)$, the refractive index $n_{\text {clad }}$ of the low-refractive index material (waveguide cladding), the refractive index $n_{\text {core }}$ of the HRI material (waveguide core):

$$
\begin{aligned}
& \tan \left(\frac{\pi}{\lambda} \cdot t \cdot n_{\text {core }} \cdot \sin \theta_{m}-m \frac{\pi}{2}\right) \\
& \quad= \begin{cases}\frac{\sqrt{n_{\text {core }}^{2} \cdot \cos ^{2} \theta_{m}-n_{\text {clad }}^{2}}}{n_{\text {core }} \cdot \sin \theta_{m}} & \text { for TE modes } \\
\frac{n_{\text {core }} \sqrt{n_{\text {core }}^{2} \cdot \cos ^{2} \theta_{m}-n_{\text {clad }}^{2}}}{n_{\text {clad }}^{2} \cdot \sin \theta_{m}} & \text { for TM modes }\end{cases}
\end{aligned}
$$

where $\theta_{m}$ is the internal angle of propagation in the slab waveguide and $m$ is the waveguide propagation order. The 
angle $\theta_{m}$ is related to the in-coupling and out-coupling angles through the grating equation:

$$
\begin{aligned}
& \left\{\begin{array}{l}
k_{x_{\text {in }, \text { out }}}=\frac{2 \pi}{\lambda} \cdot n_{\text {clad }} \cdot \sin \theta_{\text {in }, \text { out }} \cdot \cos \phi_{\text {in }, \text { out }}-i \frac{2 \pi}{\Lambda_{1,2}} \\
k_{y_{\text {in, out }}}=\frac{2 \pi}{\lambda} \cdot n_{\text {clad }} \cdot \sin \theta_{\text {in }, \text { out }} \cdot \sin \phi_{\text {in }, \text { out }}
\end{array}\right. \\
& \rightarrow\left\{\begin{array}{l}
\theta_{m}=\tan ^{-1} \frac{\sqrt{k_{x_{\mathrm{in}, \text { out }}}^{2}+k_{y_{\mathrm{in}, \text { out }}}^{2}}}{k_{z_{\text {in out }}}} \\
\phi_{m}=\tan ^{-1} \frac{k_{y_{\text {in,out }}}}{k_{z_{\text {in out }}}}
\end{array}\right.
\end{aligned}
$$

The angle $\theta_{m}$ has to be the same for both the in-coupled and out-coupled waves in order to respect the impedance-matching condition between both RWGs building the unit cell. To enhance the efficiency of this unit cell and to work with substantially subwavelengths thickness structures, we set $i= \pm 1$. Furthermore, we use the fundamental waveguide mode $m=0$, such that a very thin waveguide layer can be used and supports only one propagating mode, thus, enhancing the color-purity of the device.

An infinite-long RWG would filter the spectrum of the leaky mode toward monochromatic RWG modes. For finite RWGs, the bandwidth of the in-coupled spectrum, as well as the angular tolerances, depend on the number of grooves ${ }^{37}$ and are not limited to this central wavelength.

Figure 2 shows the spatial dispersion graph for a typical unit cell for TE polarization, where the incoming light is at in-plane

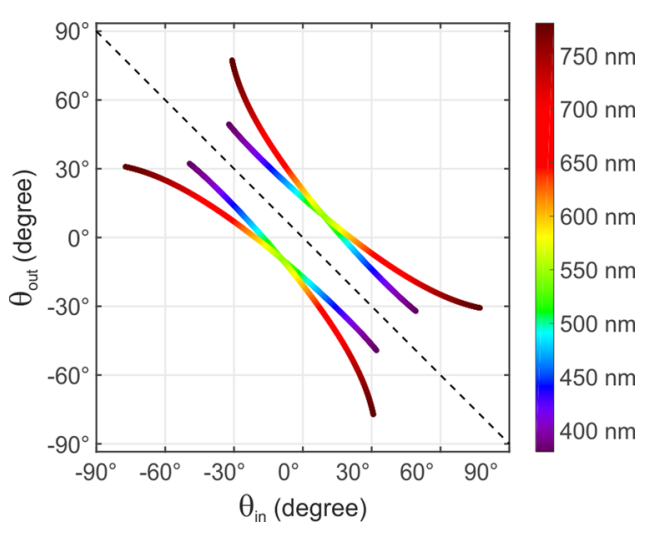

Figure 2. Spatial dispersion graph obtained with the analytical model (eqs 1,2$)$ for a typical unit cell $\left(\Lambda_{1}=380 \mathrm{~nm}, \Lambda_{2}=310 \mathrm{~nm}, t=24 \mathrm{~nm}\right.$, zinc sulfide as HRI material, Ormocomp as cladding material, $\phi_{\text {in }}=$ $\phi_{\text {out }}=0^{\circ}$ ) showing the in-coupling $\theta_{\text {in }}$ and the out-coupling $\theta_{\text {out }}$ angles. It is possible to in-couple the light from both RWGs and from both diffracted orders that can be supported $(i=+1)$ by each RWG.

incidence (i.e., $\phi_{\text {in }}=0$ ). When we change the polar angle of incidence (i.e., in-coupling angle $\theta_{\text {in }}$ ) we observe a variation of the out-coupled light, both in its spectrum and direction. We notice in Figure 2 up to four different peaks (in the visible spectrum for this configuration). Indeed, it is possible to incouple light from each of the two gratings and from both the $i=$ \pm 1 and the $i=-1$ diffracted orders. We notice that the spatial dispersion lines form pairs of symmetric lines with respect to the $\theta_{\text {in }}-\theta_{\text {out }}$ axis. This symmetry is related to the $k$-space symmetry of the system, when both gratings are exchanged and illuminated from the opposite angle $-\theta_{\text {in }}$. Let us note that the data reported in Figure 2 are valid both in reflection and transmission. Some simulations showing the efficiencies of those resonances as a function of wavelength and angle are shown in section S4 of the Supporting Information, showing the stability of the efficiencies for different colors.

In order to evaluate this analytical model, we first evaluate the optical properties of the unit cell with finite-difference timedomain (FDTD) simulations. A representation of the TE electric near field in the collinear plane in a stationary state is shown in Figure 1c,d. The unit cell lengths are $L_{1}=L_{2}=25 \mu \mathrm{m}$, while their width is $100 \mu \mathrm{m}$.

As illustrated in Figure 1e, we have engineered a planar optics surface composed of different unit cells that can be illuminated with the flash of a smartphone as broadband source and observed with the back-camera of this smartphone. To achieve constructive interference in any illumination coherence of the plurality of steered light beams at the observer, the distance between different unit cells $s$ has been tuned such that the path difference from the source to the observer is a multiple of the central steered wavelength $\lambda$ :

$$
r_{\mathrm{SD}}^{\prime}-r_{\mathrm{SD}}=n \lambda
$$

where $n$ is an integer, and $r_{\mathrm{SD}}^{\prime}$ and $r_{\mathrm{SD}}$ are the paths from the source to the observer passing through the center of different unit cells. We evaluate the optical path considering the center of the unit cells since $L_{1}=L_{2}$.

The fabrication of the device (Figure $2 a-d$ ) is explained in the Methods section, where a SEM image and an AFM profile (Figure 2e,f) are also explained. Due to the limited number of process steps, the structure can be easily replicated using wafer to wafer, sheet to sheet and roll-to-roll NIL, which are suitable for industrial applications with a high-throughput.

We designed and fabricated a specific unit cell to characterize the efficiency of the structure. In this device, we set all the unit cells to have the same periods $\left(\Lambda_{1}=380 \mathrm{~nm}\right.$ and $\Lambda_{2}=$ $310 \mathrm{~nm})$, all aligned into the same direction and with no separation between them $\left(s=L_{1}+L_{2}=50 \mu \mathrm{m}\right)$. A FDTD simulation of this structure was performed and the far-field results are plot in Figure 4a. As shown in section S3 of the Supporting Information, the four resonances are similar in both transmission and reflection. As reflectance measurements cannot be performed at near-normal incidence, we chose to characterize the transmittance.

Figure $4 c$ shows the experimental results, where we observe four resonances similar to those obtained in the simulations. The differences, especially in the angular spreading of the resonances, are mainly related to the optical setup (in particular to a $0.2^{\circ}$ beam divergence of the light source and the $0.5^{\circ}$ numerical aperture of the spectrometer) and to the fabricated device, whose profile differs from the simulated one because of more sinusoidal corrugations. These resonances represent the four possible coupled modes obtained in the analytical model (Figure $4 \mathrm{~b}$ ). The analytical model reproduces very well the experimental and full-field numerical data, in spite of the important corrugation of the waveguide (let us recall that eq 1 holds for a smooth waveguide). Figure $4 \mathrm{~d}$ indicates the bandwidth of the resonances, which is $14 \mathrm{~nm}$ for all the resonances, compared with the simulations. This bandwidth is well suited for the optical security application described below, and it can be reduced or increased by varying the length of the unit cell (i.e., $L_{1}, L_{2}$ in Figure 1). ${ }^{37}$ The angular spread is about 
a

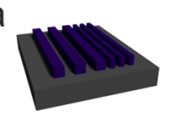

C

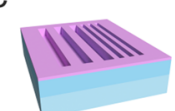

b
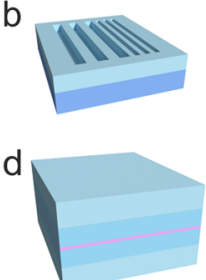
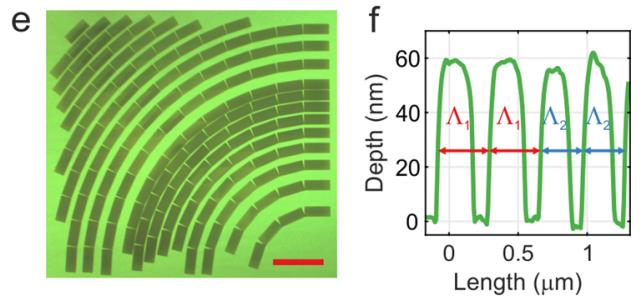

Figure 3. Process flow for the fabrication of the device. (a) The structure is created by e-beam lithography on a Si-wafer with a layer of the negative photoresist HSQ that is then developed in TMAH. (b) It is possible to replicate that structure by UV-NIL lithography on a glass-substrate using a curable sol-gel material. (c) Guiding layer of the structure is coated with PVD. (d) The device is finally encapsulated with a UV-curable sol-gel and another cover glass. (e) Optical microscope images of a typical pattern, each visible rectangle is a unit cell comprising two coupled RWGs (scale bar $200 \mu \mathrm{m}$ ). (f) AFM profile of the center of a unit cell composed of two RWGs with different periods replicated using UV-NIL.
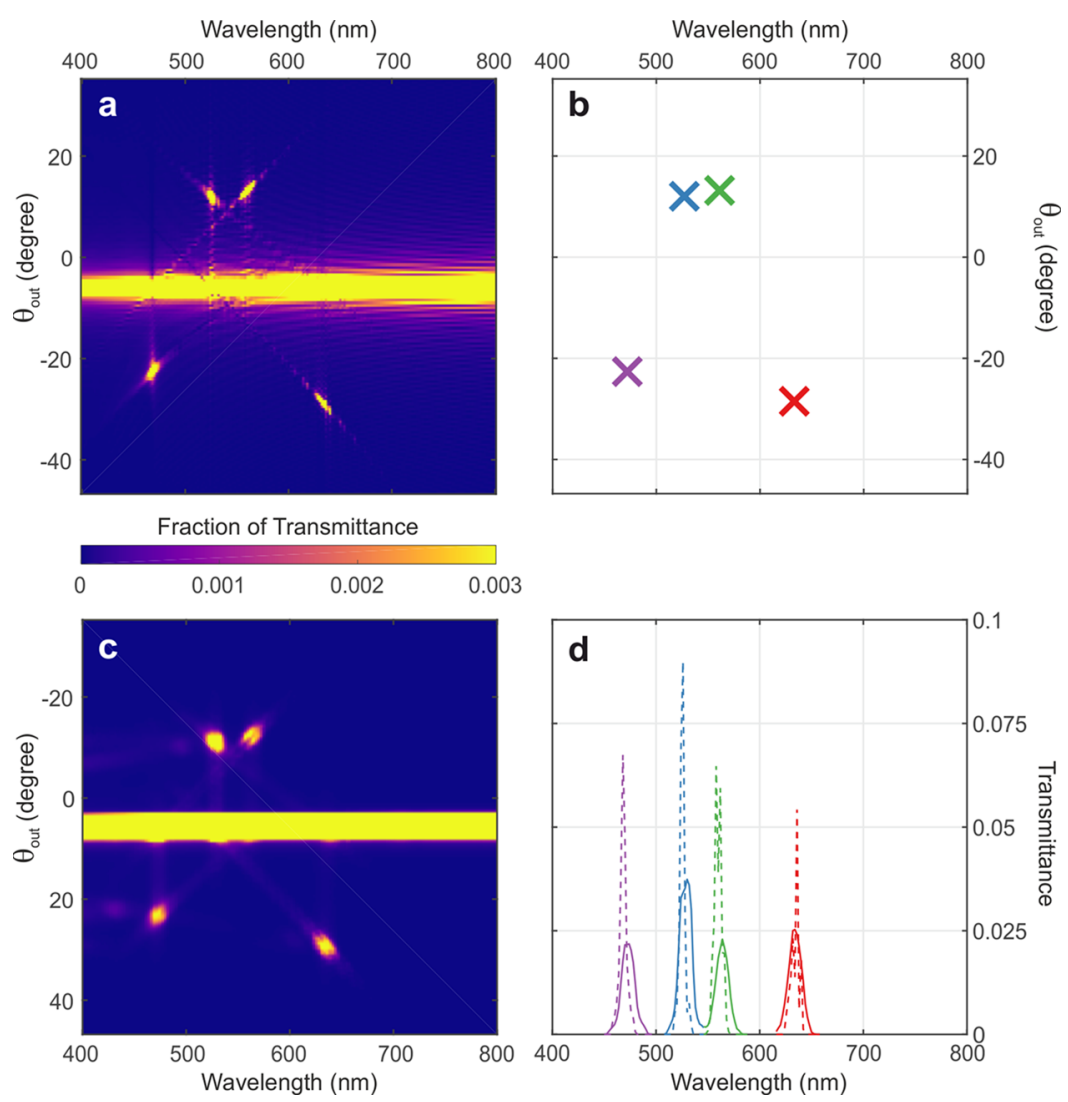

Figure 4. (a) Simulations and (c) measurements of the transmittance integrated every $0.5^{\circ}$ for an array of unit cells with two adjacent RWGs $\left(\Lambda_{1}=\right.$ $380 \mathrm{~nm}, \Lambda_{2}=310 \mathrm{~nm}, t=24 \mathrm{~nm}$, zinc sulfide as HRI material, Ormocomp as cladding material, $\phi_{\text {in }}=\phi_{\text {out }}=0^{\circ}, \theta_{\text {in }}=4^{\circ}$ ), where the illumination is TE polarized and tilted by $6^{\circ}$, normalized to the illumination in air. (b) The comparison with the analytical model. (d) The transmittance integrated over the angular spread of the four peaks is compared between measurements (solid lines) and simulations (dashed lines).

$3^{\circ}$ for each resonance, and it can be reduced by engineering the distance between different unit cells (eq 3). The efficiency of each resonance is reasonably smaller than $10 \%$ because of the following considerations: the in-coupling grating covers about $50 \%$ of the area, there is an efficiency of about $50 \%$ in both the in-coupling and out-coupling processes in leaky RWGs, and the out-coupled light is split approximately in equal ratio in both the superstrate and the substrate. With these assumptions, we expect less than $10 \%$ of efficiency for each resonance.

To verify the possibility of achieving constructive interferences as illustrated in Figure 1e, we have fabricated another test device with all the unit cells aligned. In this case, however, the unit cells have different distances from each other and different grating periods, engineered in a way such to create constructive interference in a specific point. This device is explained in greater details in Figure S1 of the Supporting Information.

In Figure 5, two different realizations of a device for smartphone are shown. These samples have been made for optical security applications and they have been designed to work with a standard smartphone, using the design approach of Figure 1e, where $f$ is the distance between the flash and the camera. It is possible to see the desired pattern only for a specific smartphone location, where the entire pattern exhibits a constructive interference. The effect is obtained only for the order $i=-1$ for the in-coupling process and the order $i=+1$ for the out-coupling one, while the other channel does not yield constructive interference. Under normal ambient light condition, the structure is almost completely transparent and it is not possible to observe any diffraction by naked eye, except at 
a

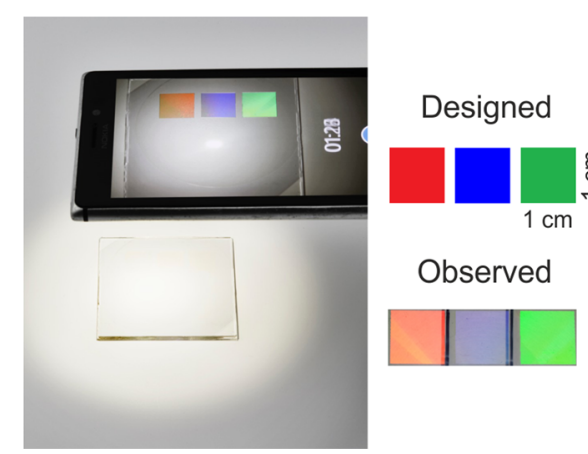

b

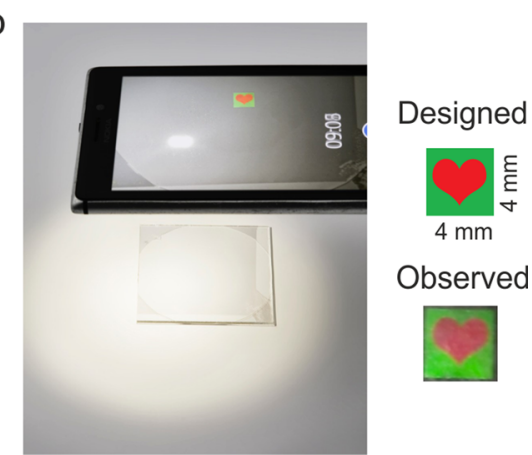

Figure 5. Images of two devices created with a customized pattern where the source and the observer are the flash and the camera of a smartphone. Note that the devices are not visible outside the designed observation point. (a) The first realization comprises uniform red, blue, and green squares $\left(1 \times 1 \mathrm{~cm}^{2}\right.$ each); (b) The second realization represents a red heart with green background $\left(0.4 \times 0.4 \mathrm{~cm}^{2}\right)$.

very large angles. Thanks to the tolerance of the configuration (e.g., out-coupled spectrum of $14 \mathrm{~nm}$ and broad steering angle, flash, and camera not being point light source and observer), the effect is robust with respect to the smartphone positioning. In particular, it accepts some offset in all the three directions, as well as in the distance between source and observer. In fact, the effect has been tested with different smartphones (e.g., Nokia Lumia 920, Samsung Galaxy S3, and iPhone 6s) and it correctly appeared as designed. The tenuous color variations between the designed and the observed colors are mostly related to the smartphone hardware and the postprocessing software (i.e., the spectrum of the LED, the characteristics of the CMOS sensor, the brightness level, as well as the white balance).

In summary, we have demonstrated a beam steering planar optics structure that selectively filters and redirects spectral portions of the incoming light. It operates under unpolarized white light and low-coherence source and works both in transmission and reflection. This device relies on unit cells that are designed by choosing a particular pair of impedancematched RWGs, where the first acts as in-coupler and the second acts as out-coupler. This structure exhibits a good color tunability; it is made by only one NIL replication and one thin film layer deposition, such that its fabrication is compatible with up-scalable fabrication processes. The demonstrated device is therefore well-suited for industrial applications, where high throughput is required. Further developments can include the possibility of using ultrathin metallic waveguides or combined metallic/dielectric waveguides. The reported concept was created for an optical security application, for which RWGs have been very successful and are used to secure billion of documents every year. ${ }^{32}$ It is also possible to take advantage of the flexibility and the versatility of the structure for a variety of other applications where beam steering is required, such as multifocal or monochromatic lenses, ${ }^{42}$ biosensors, ${ }^{43}$ and seethrough optical combiners for near-eye displays, ${ }^{44}$ to name a few.

\section{METHODS}

Simulations. 2D FDTD simulations were performed using the commercial software OmniSim distributed by Photon Design and Lumerical FDTD distributed by Lumerical Solutions. To compute the plot shown in Figure $4 b$, a single unit cell was modeled and Bloch boundary conditions were used for defining the periodicity of the unit cell in the $x$ direction, while PML boundary conditions were used in the $z$ direction below the substrate and above the superstrate. The typical mesh size used in the calculation was $7 \times 12 \mathrm{~nm}^{2}$. To simulate a broadband spectrum with Bloch boundary conditions, we performed one simulation of the structure for every wavelength (TE polarization). In order to include the losses at the interface air/cladding material, the data were postprocessed with a transfer matrix computation. ${ }^{45}$ Far-field analysis was adjusted to obtain the angular distribution of the electric field.

Sample Fabrication. The fabrication of the device involves e-beam lithography and nanoimprint lithography (NIL) processes. The master for the structure is made by exposing HSQ photoresist (HSQ XR1541 004) on a Si wafer using ebeam lithography (EBPG5000ES). A SEM picture of a typical pattern after ebeam lithography is shown in Figure $3 \mathrm{e}$. Afterward, the resist is developed in a TMAH solution (Figure 3a). Using UV-NIL lithography, the device is replicated on a glass using a UV curable sol-gel and hard-backed (Figure 3b). An AFM profile of the structure after this step is shown in Figure 3f. Subsequently, the $\mathrm{ZnS}$ layer is deposited with PVD (Figure 3c), and finally, the structure is encapsulated by the same UV sol-gel used for the NIL lithography (Figure 3d). See Supporting Information, section S2 for the corresponding material parameters.

Characterization. A broad-spectrum white light covering the whole visible and near-IR spectrum (400-1000 nm) was generated with a Halogen Lamp (HL-2000, Ocean Optics) and in-coupled into a fiber with NA $=0.15$. The fiber was connected to a parabolic reflective collimator (RC04SMA, Thorlabs) and reduced in diameter with an aperture $(3 \mathrm{~mm})$. The system was placed on a motorized rotation stage (CR1-Z7, Thorlabs) to scan the angle of transmission. A second motorized rotation stage was placed on top of the previous one. The sample was placed on that second stage with a holder in order to change the incidence angle of the collimated light on the sample. The whole system was then placed on a motorized linear stage (LTS300, Thorlabs) to achieve a higher precision of alignment. A spectroradiometer (Spectrascan PR730, Photo Research) was used to measure the transmitted light after the sample. The transmitted light was recorded from $-5^{\circ}$ to $40^{\circ}$ with steps of $0.5^{\circ}$ with a numerical aperture of the spectroradiometer equivalent to $0.5^{\circ}$. The data were then normalized to air.

Patterns Design. The patterns required for the devices shown in Figure 5 were generated with a Matlab script using eqs $1-3$, assuming $\lambda_{\text {red }}=630 \mathrm{~nm}, \lambda_{\text {green }}=535 \mathrm{~nm}, \lambda_{\text {blue }}=$ $480 \mathrm{~nm}, t=70 \mathrm{~nm}, f=10 \mathrm{~nm}$ and the vertical distance between 
the source/observer and the structure was $65 \mathrm{~nm}$. The cladding material was set to be Ormocomp and the HRI material used was $\mathrm{ZnS}$ (see section S2 in the Supporting Information for the corresponding materials parameters).

\section{ASSOCIATED CONTENT}

\section{S Supporting Information}

The Supporting Information is available free of charge on the ACS Publications website at DOI: 10.1021/acsphotonics.7b00232.

Optical response of a pattern created to provide constructive interference in a specific point; refractive index of the materials; comparison between simulations in transmission and reflection; efficiencies of resonances as a function of wavelength and angle; description of the videos (PDF).

Video of a simulation showing the $E_{\mathrm{y}}$ amplitude in steady state with $\Lambda_{2}=380 \mathrm{~nm}$ (AVI).

Video of a simulation showing the $E_{\mathrm{y}}$ amplitude in steady state with $\Lambda_{2}=450 \mathrm{~nm}$ (AVI).

Video of a simulation showing the $E_{\mathrm{y}}$ amplitude in steady state with $\Lambda_{2}=200 \mathrm{~nm}$ (AVI).

Video of a fabricated device comprising red, blue, and green squares (AVI).

Video of a fabricated device comprising an animation (AVI)

Video of a fabricated device comprising a white square (AVI)

\section{AUTHOR INFORMATION}

\section{Corresponding Author}

*E-mail: gba@csem.ch. Tel.: +41616906044.

\section{ORCID}

Giorgio Quaranta: 0000-0003-3471-3802

Olivier J. F. Martin: 0000-0002-9574-3119

Notes

The authors declare no competing financial interest.

\section{ACKNOWLEDGMENTS}

We are grateful to Dr. Zdenek Benes for his precious advices for optimizing the ebeam lithography process. O.J.F.M. acknowledges funding from the European Research Council (ERC-2015-AdG-695206 Nanofactory).

\section{REFERENCES}

(1) Vasey, F.; Reinhart, F. K.; Houdre, R.; Stauffer, J. M. Spatial Optical Beam Steering with an AlGaAs Integrated Phased Array. Appl. Opt. 1993, 32, 3220-3232.

(2) Holz, M.; Goltsos, W. C. Agile Beam Steering Using Binary Optics Microlens Arrays. Opt. Eng. 1990, 29, 1392-1397.

(3) Völkel, R. Microlens Array Imaging System for Photolithography. Opt. Eng. 1996, 35, 3323.

(4) Kunz, R. E. Gradient Effective Index Waveguide Sensors. Sens. Actuators, B 1993, 11, 167-176.

(5) Fattinger, C. P.; Mangold, C.; Gale, M. T.; Schuetz, H. Bidiffractive Grating Coupler: Universal Transducer for Optical Interface Analytics. Opt. Eng. 1995, 34, 2744-2753.

(6) Cottier, K.; Wiki, M.; Voirin, G.; Gao, H.; Kunz, R. E. Label-Free Highly Sensitive Detection of (Small) Molecules by Wavelength Interrogation of Integrated Optical Chips. Sens. Actuators, B 2003, 91, 241-251.
(7) Davoine, L.; Paeder, V.; Basset, G.; Schnieper, M.; Herzig, H. P. Resonant Absorption of a Chemically Sensitive Layer Based on Waveguide Gratings. Appl. Opt. 2013, 52, 340-349.

(8) Parriaux, O.; Voirin, G. Plasmon Wave versus Dielectric Waveguiding for Surface Wave Sensing. Sens. Actuators, A 1990, 23, $1137-1141$.

(9) Taghizadeh, M. R.; Blair, P.; Layet, B.; Barton, I. M.; Waddie, A. J.; Ross, N. Design and Fabrication of Diffractive Optical Elements. Microelectron. Eng. 1997, 34, 219-242.

(10) Yu, N.; Genevet, P.; Kats, M. A.; Aieta, F.; Tetienne, J.-P.; Capasso, F.; Gaburro, Z. Light Propagation with Phase Discontinuities: Generalized Laws of Reflection and Refraction. Science 2011, 334, 333-337.

(11) Kildishev, A. V.; Boltasseva, A.; Shalaev, V. M. Planar Photonics with Metasurfaces. Science 2013, 339, 1232009-1232009.

(12) Verslegers, L.; Catrysse, P. B.; Yu, Z.; White, J. S.; Barnard, E. S.; Brongersma, M. L.; Fan, S. Planar Lenses Based on Nanoscale Slit Arrays in a Metallic Film. Nano Lett. 2009, 9, 235-238.

(13) Fattal, D.; Li, J.; Peng, Z.; Fiorentino, M.; Beausoleil, R. G. Flat Dielectric Grating Reflectors with Focusing Abilities. Nat. Photonics 2010, 4, 466-470.

(14) Aieta, F.; Genevet, P.; Kats, M. A.; Yu, N.; Blanchard, R.; Gaburro, Z.; Capasso, F. Aberration-Free Ultrathin Flat Lenses and Axicons at Telecom Wavelengths Based on Plasmonic Metasurfaces. Nano Lett. 2012, 12, 4932-4936.

(15) Genevet, P.; Yu, N.; Aieta, F.; Lin, J.; Kats, M. A.; Blanchard, R.; Scully, M. O.; Gaburro, Z.; Capasso, F. Ultra-Thin Plasmonic Optical Vortex Plate Based on Phase Discontinuities. Appl. Phys. Lett. 2012, $100,013101$.

(16) West, P. R.; Stewart, J. L.; Kildishev, A. V.; Shalaev, V. M.; Shkunov, V. V.; Strohkendl, F.; Zakharenkov, Y. A.; Dodds, R. K.; Byren, R. All-Dielectric Subwavelength Metasurface Focusing Lens. Opt. Express 2014, 22, 26212.

(17) Aieta, F.; Kats, M. A.; Genevet, P.; Capasso, F. Multiwavelength Achromatic Metasurfaces by Dispersive Phase Compensation. Science 2015, 347, 1342-1345.

(18) Pors, A.; Nielsen, M. G.; Eriksen, R. L.; Bozhevolnyi, S. I. Broadband Focusing Flat Mirrors Based on Plasmonic Gradient Metasurfaces. Nano Lett. 2013, 13, 829-834.

(19) Khorasaninejad, M.; Aieta, F.; Kanhaiya, P.; Kats, M. A.; Genevet, P.; Rousso, D.; Capasso, F. Achromatic Metasurface Lens at Telecommunication Wavelengths. Nano Lett. 2015, 15, 5358-5362.

(20) Genevet, P.; Capasso, F.; Aieta, F.; Khorasaninejad, M.; Devlin, R. Recent Advances in Planar Optics: From Plasmonic to Dielectric Metasurfaces. Optica 2017, 4, 139.

(21) Lin, D.; Fan, P.; Hasman, E.; Brongersma, M. L. Dielectric Gradient Metasurface Optical Elements. Science 2014, 345, 298-302.

(22) Yu, Y. F.; Zhu, A. Y.; Paniagua-Domínguez, R.; Fu, Y. H.; Luk'yanchuk, B.; Kuznetsov, A. I. High-Transmission Dielectric Metasurface with $2 \pi$ Phase Control at Visible Wavelengths: HighTransmission Dielectric Metasurface with $2 \pi$ Phase Control at Visible Wavelengths. Laser Photonics Rev. 2015, 9, 412-418.

(23) Khorasaninejad, M.; Chen, W. T.; Devlin, R. C.; Oh, J.; Zhu, A. Y.; Capasso, F. Metalenses at Visible Wavelengths: Diffraction-Limited Focusing and Subwavelength Resolution Imaging. Science 2016, 352, $1190-1194$.

(24) Chen, X.; Huang, L.; Mühlenbernd, H.; Li, G.; Bai, B.; Tan, Q.; Jin, G.; Qiu, C.-W.; Zhang, S.; Zentgraf, T. Dual-Polarity Plasmonic Metalens for Visible Light. Nat. Commun. 2012, 3, 1198.

(25) Gutruf, P.; Zou, C.; Withayachumnankul, W.; Bhaskaran, M.; Sriram, S.; Fumeaux, C. Mechanically Tunable Dielectric Resonator Metasurfaces at Visible Frequencies. ACS Nano 2016, 10, 133-141.

(26) Wang, B.; Dong, F.; Li, Q.-T.; Yang, D.; Sun, C.; Chen, J.; Song, Z.; Xu, L.; Chu, W.; Xiao, Y.-F.; Gong, Q.; Li, Y. Visible-Frequency Dielectric Metasurfaces for Multiwavelength Achromatic and Highly Dispersive Holograms. Nano Lett. 2016, 16, 5235-5240.

(27) Sturmberg, B. C. P.; Chong, T. K.; Choi, D.-Y.; White, T. P.; Botten, L. C.; Dossou, K. B.; Poulton, C. G.; Catchpole, K. R.; McPhedran, R. C.; Martijn de Sterke, C. Total Absorption of Visible 
Light in Ultrathin Weakly Absorbing Semiconductor Gratings. Optica

2016, 3, 556.

(28) Sell, D.; Yang, J.; Doshay, S.; Zhang, K.; Fan, J. A. Visible Light Metasurfaces Based on Single-Crystal Silicon. ACS Photonics 2016, 3, 1919.

(29) Ahn, S. H.; Guo, L. J. Large-Area Roll-to-Roll and Roll-to-Plate Nanoimprint Lithography: A Step toward High-Throughput Application of Continuous Nanoimprinting. ACS Nano 2009, 3, 2304-2310.

(30) Gale, M.; Knop, K.; Morf, R. Zero-Order Diffractive Microstructures for Security Applications; Fagan, W., Ed.; Spie-Int Soc Optical Engineering: Bellingham, 1990; Vol. 1210.

(31) Liu, Q.; Duan, X.; Peng, C. Mass Production of Large-Format Micro-/Nanostructure-Based Optical Devices. In Novel Optical Technologies for Nanofabrication; Springer: Berlin, Heidelberg, 2014; pp 223-267.

(32) Surys DID. http://surys.com/surys-optical-sciences-did/ (accessed Mar 9, 2017).

(33) Wang, S. S.; Moharam, M. G.; Magnusson, R.; Bagby, J. S. Guided-Mode Resonances in Planar Dielectric-Layer Diffraction Gratings. J. Opt. Soc. Am. A 1990, 7, 1470-1474.

(34) Wang, S. S.; Magnusson, R. Theory and Applications of GuidedMode Resonance Filters. Appl. Opt. 1993, 32, 2606-2613.

(35) Rosenblatt, D.; Sharon, A.; Friesem, A. A. Resonant Grating Waveguide Structures. IEEE J. Quantum Electron. 1997, 33, 20382059.

(36) Avrutsky, I.; Rabady, R. Waveguide Grating Mirror for LargeArea Semiconductor Lasers. Opt. Lett. 2001, 26, 989-991.

(37) Boye, R. R; Kostuk, R. K. Investigation of the Effect of Finite Grating Size on the Performance of Guided-Mode Resonance Filters. Appl. Opt. 2000, 39, 3649.

(38) Palmer, C. A.; Loewen, E. G. Diffraction Grating Handbook; Newport Corporation: Springfield, Ohio, U.S.A., 2005.

(39) Prokhorov, A. M.; Spikhal'skiǐ. Emission of E and H Waves from a Corrugated Section of a Diffused Waveguide. Soviet J. Quantum Electronics 1976, 6, 10.

(40) Zlenko, A. A.; Prokhorov, A. M.; Spikhal'skiĭ, A. A.; Sychugov, V. A. Emission of E Waves from a Corrugated Section of a Waveguide. Sov. J. Quantum Electron. 1976, 6, 565.

(41) Okamoto, K. Fundamentals of Optical Waveguides; Academic Press, 2010.

(42) Chen, H.-T.; Taylor, A. J.; Yu, N. A Review of Metasurfaces: Physics and Applications. Rep. Prog. Phys. 2016, 79, 076401.

(43) Daghestani, H. N.; Day, B. W. Theory and Applications of Surface Plasmon Resonance, Resonant Mirror, Resonant Waveguide Grating, and Dual Polarization Interferometry Biosensors. Sensors 2010, 10, 9630-9646.

(44) Kress, B.; Starner, T. A Review of Head-Mounted Displays (HMD) Technologies and Applications for Consumer Electronics. Proc. SPIE 2013, 8720, 87200A.

(45) Junesch, J.; Sannomiya, T.; Dahlin, A. B. Optical Properties of Nanohole Arrays in Metal-Dielectric Double Films Prepared by Mask-on-Metal Colloidal Lithography. ACS Nano 2012, 6, 1040510415. 